\title{
The Liar Without Relativism
}

\section{Poppy Mankowitz ${ }^{1}$}

Received: 3 February 2020/Accepted: 14 December 2020/Published online: 19 March 2021 (C) The Author(s) 2021

\begin{abstract}
Some in the recent literature have claimed that a connection exists between the Liar paradox and semantic relativism: the view that the truth values of certain occurrences of sentences depend on the contexts at which they are assessed. Sagi (Erkenntnis 82(4):913-928, 2017) argues that contextualist accounts of the Liar paradox are committed to relativism, and Rudnicki and Łukowski (Synthese 1-20, 2019) propose a new account that they classify as relativist. I argue that a full understanding of how relativism is conceived within theories of natural language shows that neither of the purported connections can be maintained. There is no reason why a solution to the Liar paradox needs to accept relativism.
\end{abstract}

\section{Introduction}

The Liar sentence $L$, which reads ' $L$ is not true', can be used to produce an apparently valid argument proving that $L$ is not true (or fails to express a proposition, or fails to express a true proposition) and that $L$ is true (or expresses a proposition, or expresses a true proposition). This is the Liar paradox, and it appears to be the 'symptom of a major problem with our reasoning about truth' (Scharp 2013 b, 429). Numerous analyses seek to show that no genuine contradiction can be derived from the Liar sentence (e.g., Chihara 1979; Gupta 1982; Maudlin 2004; McGee 1991; Scharp 2013a). Contextualist accounts comprise a popular solution, arguing that some step in the reasoning induces a context shift, and the apparently contradictory claims thus occur and hold at different contexts (see Barwise and Etchemendy 1987; Burge 1982; Glanzberg 2001; Murzi and Rossi 2018; Parsons 1974; Simmons 1993). The thesis that the truth values of certain occurrences of sentences depend on the contexts in which they are used is uncontroversial in linguistics and the philosophy of language.

Poppy Mankowitz

poppy.mankowitz@bristol.ac.uk

1 University of Bristol, Bristol, UK 
Some in the recent literature have drawn a connection between solutions to the Liar paradox and relativism, a more controversial thesis holding that the truth values of occurrences of sentences also depend on the contexts at which they are assessed. Sagi (2017) purports to show that contextualist accounts are committed to relativism about the Liar sentence. It would follow that such accounts have been 'mischaracterised and [...] should be re-evaluated according to their full implications' (Sagi $2017,913)$. One implication is that contextualist accounts would be vulnerable to criticisms of relativism advanced in the literature, where some such criticisms have been advanced by contextualists themselves (see Glanzberg 2007). Rudnicki and Eukowski (2019) present experimental evidence that they take to support an analysis of the Liar sentence as necessarily false, a position that they classify as a form of relativism. It would follow that all existing accounts must be reconciled with empirical data that support a relativist analysis of the Liar sentence. Furthermore, empirical evidence for a relativist position would have ramifications for ongoing debates within linguistics about whether any natural language expressions are sensitive to contexts of assessment. In the current paper, I argue that a complete understanding of relativism shows that neither of the purported connections can be maintained.

In Sect. 2, I provide an overview of contextualism and relativism. Section 3 sets out Sagi's argument for contextualists' commitment to relativism, before presenting several objections. Section 4 describes then opposes Rudnicki and Łukowski's proposal and their reason for relating it to relativism. Section 5 challenges an additional independent argument for connecting relativism and the Liar. I conclude that there is no need for a solution to the Liar paradox to accept relativism.

\section{Relativism}

In Sect. 2.1, I explain how contextualism and relativism are characterised within linguistics and the philosophy of language. Section 2.2 clarifies the implications of a relativist analysis of the Liar sentence.

\subsection{Relativism and Contextualism}

It is obvious that contexts play a role in determining the truth values of natural language sentences. For example, the sentence 'I am walking' cannot sensibly be described as 'true' or 'false' simpliciter. Rather, it can only be assigned a truth value relative to particular contexts. It can be assigned the value true if and only if it is uttered by an agent who is walking at the time of utterance. To capture the influence of contexts on truth values, contexts must accordingly be thought of as determining at least a unique world, time, location and agent; though they are often thought to determine additional features, such as standards of taste and bodies of information.

Sensitivity of semantic values to contexts of use is assumed by standard analyses of expressions like indexicals and demonstratives ('I', 'here', 'now', 'that', etc.; see Kaplan 1989), gradable adjectives ('tall', 'rich', etc.; see Kennedy and McNally 2005), modals ('might', 'ought', 'possible', etc.; see Kratzer 1981), and quantifier 
expressions ('every', 'most', etc.; see Stanley and Szabó 2000). Yet it has been argued that the truth values of certain types of sentences also depend on contexts of assessment, which are 'possible situation[s] in which a speech act or mental attitude might be assessed' (MacFarlane 2012, 135). This position is often motivated by observing that the natural way of evaluating a given type of sentence appears incompatible with its having a semantic value fixed by its context of use. ${ }^{1}$ It has been argued that contexts of assessment affect the truth values of occurrences of sentences containing predicates of personal taste (e.g., 'Rollercoasters are fun'; see Lasersohn 2005, 2009; MacFarlane 2005b; Stephenson 2007), epistemic modals (e.g., 'It might be raining outside'; Egan 2007; Egan et al. 2005; MacFarlane 2011a; Stephenson 2007), deontic modals (e.g., 'We ought to save the drowning child'; Kolodny and MacFarlane 2010), knowledge claims (e.g., 'I know that it is raining'; MacFarlane 2005a, 2011b; Richard 2004) and future contingents (e.g., 'It will rain tomorrow'; MacFarlane 2003, 2008).

To demonstrate the coherence of relativism, and to clarify how it differs from contextualism, an account is required of the ways in which contexts of use and contexts of assessment can affect the interpretation of expressions. The most detailed account is provided by MacFarlane (2003, 2005b, 2007, 2011a, 2012, 2014). He first distinguishes between the following forms of context sensitivity (e.g., in 2014, 64):

Use sensitive (MacFarlane) An expression is use sensitive iff its extension (relative to a context of use and context of assessment) depends on features of the context of use.

Assessment sensitive (MacFarlane) An expression is assessment sensitive iff its extension (relative to a context of use and context of assessment) depends on features of the context of assessment.

He then defines a relativist semantic theory as one that takes some expressions to be assessment sensitive (MacFarlane 2012, 135), whereas a contextualist semantic theory only attributes use sensitivity to expressions.

A complication emerges from the fact that standard semantic frameworks-such as the one developed in Kaplan 1989-include no means by which features of a context of assessment could affect the semantic values of expressions. Kaplan $(1989,502)$ describes circumstances of evaluation as 'actual and counterfactual scenarios with respect to which it is appropriate to ask for the extensions of a given well-formed expression', and which include a possible world, a time, 'and perhaps

\footnotetext{
${ }^{1}$ For example, the below scenarios (from Shirreff and Weatherson 2017, 793-794) suggest a difference between personal taste predicates like 'delicious' and expressions like 'pleasant here':

1. $\operatorname{Kim}($ age 8): Lunchables are delicious.

Kim (age 27, reminded of previous assertion): I take it back / What I said was false.

2. Kim (in a cafe): It is pleasant here.

Kim (in an oil refinery, reminded of previous assertion): ? I take it back / What I said was false.
}

If the truth of an occurrence of a sentence is fixed by features of its context of use, then a speaker should not later classify the original occurrence of the sentence as false or retract it, a prediction that holds for (2) but not for (1). 
other features as well'. In this framework, a sentence expresses content (specifically, a proposition) relative to a context, and this content receives a truth value relative to a circumstance of evaluation fixed by the context. MacFarlane adapts this standard framework in two ways. First, he allows circumstances of evaluation to include features additional to worlds and times, such as tastes and bodies of information (MacFarlane 2014, 82-8). Second, he assumes that it is not only contexts of use that may affect contents and circumstances of evaluation, but also contexts of assessment. According to the resulting picture, an expression may be use (assessment) sensitive because its content is determined by some feature $F$ of the context of use (assessment), or because the circumstance at which it is evaluated includes some $F$ of the context of use (assessment). ${ }^{2}$

For instance, a contextualist about 'delicious' might hold that 'Liquorice is delicious' expresses different propositions at different contexts of use (say, when used at $c_{i}$ it expresses the proposition that liquorice is pleasing to the tastes at $c_{i}$ ), and a relativist might hold that it expresses different propositions at different contexts of assessment (say, when used at $c_{i}$ and assessed at $c_{j}$, it expresses the proposition that liquorice is pleasing to the tastes at $c_{j}$ ). Alternatively, a contextualist and relativist might agree that 'Liquorice is delicious' expresses the same proposition relative to each context of use and assessment (say, that liquorice is pleasing to the tastes at the circumstance of evaluation); but a contextualist would then hold that the relevant circumstance of evaluation includes the tastes of the context of use, whereas the relativist would consider it to include the tastes of the context of assessment.

\subsection{Relativism and the Liar}

In light of the significant differences between contextualism and relativism, Sagi (2017) is correct to accuse contextualists about the Liar sentence (hereafter, 'Liar contextualists') of a serious mischaracterisation of their own position should they be committed to the assessment sensitivity of the Liar sentence. Moreover, Rudnicki and Łukowski (2019) aim to provide evidence that the brain responses elicited by Liar sentences and false sentences are statistically identical, which they take to support a relativist analysis of the Liar sentence; and if they successfully achieve this aim, then advocates of any account that treats the Liar sentence as insensitive to contexts of assessment will face the burden of reconciling their account with these data. Yet endorsing a relativist analysis of the Liar sentence has broader implications. First, relativism is a more controversial thesis than contextualism, and has faced a number of criticisms. Second, the complexity surrounding the development and defence of an explicitly relativist account of the Liar paradox

\footnotetext{
${ }^{2}$ MacFarlane $(2014,88)$ distinguishes between nonindexical contextualism and indexical contextualism, which diverge respectively on whether an expression's use sensitivity arises because some $F$ of the context of use determines the circumstance at which it is to be evaluated or its content. Similarly, MacFarlane (Ibid., 73) distinguishes truth-value relativism from content relativism, which diverge respectively on whether an expression's assessment sensitivity arises because some $F$ of the context of assessment determines the circumstance at which it is to be evaluated or its content.
} 
would render it surprising if the assessment sensitivity of the Liar sentence turned out to be a simple entailment of other views. I discuss each of these factors in turn.

Given that MacFarlane's elaboration of relativism vindicates the coherence of the notion, one might expect it to be a simple empirical matter whether any natural language expressions are assessment sensitive. Yet a number of complications surround gathering and interpreting relevant empirical data (see Beddor and Egan 2018; Katz and Salerno 2017; Khoo and Knobe 2018; Knobe and Yalcin 2014). Moreover, some have argued that relativism should be rejected even if it were to receive apparent empirical support. One such argument, often presented implicitly, is that traditional contextualist accounts of a given type of expression should be upheld by default whenever possible, hence relativists about that type of expression incur the burden of showing that no version of contextualism could be empirically adequate (e.g., see Sundell 2011, 281). A more explicit version of this argument is presented by Cappelen and Hawthorne (2009), who identify a mainstream view they call 'Simplicity' that includes the thesis: 'There are propositions and they instantiate the fundamental monadic properties of truth simpliciter and falsity simpliciter' (1). They claim that Simplicity is incompatible with relativism (though MacFarlane 2011c disputes this claim), and that ' $[\mathrm{t}]$ here is a naturalness about Simplicity that puts a heavy burden on anyone who wants to reject it' (6). Criticisms of relativism have even been given by Liar contextualists when discussing independent issues (e.g., Glanzberg 2007). For these reasons, it is far from trivial to be committed to a relativist analysis of the Liar sentence, whether this commitment is inadvertent (as Sagi claims for Liar contextualists) or deliberate (in the case of Rudnicki and Łukowski).

Moreover, despite the popularity of relativist analyses of certain types of expressions, it is rare for accounts of the Liar sentence or the object-language truth predicate to endorse relativism. To my knowledge, Scharp (2013a, 2013b) advances the only existing proposal for an explicitly relativist analysis of the predicate 'true', which he takes to be motivated by semantic paradoxes like the Liar. ${ }^{3}$ It would be surprising if the position that Scharp goes to great lengths to develop and defend turned out to be a simple entailment of treating the Liar sentence as use sensitive or necessarily false. Similarly, since Scharp explicitly argues against Liar

\footnotetext{
3 Billon (2012) and Bobzien (2017) also suggest the potential of developing relativist accounts of the Liar sentence, although neither fully develops such an account. Scharp (2013a, 231) claims that the details Billon provides about his proposal are sufficient to show that it will be 'felled by the obvious revenge paradoxes'. Whether Bobzien's proposal is genuinely relativist, as defined in Sect. 2.1, is open to doubt: she postulates viewpoint-relative 'subvalues' distinct from the ordinary values true and false, such that ordinary truth values are assigned to occurrences of sentences in a manner insensitive to contexts of assessment (see 2017, 85-6), and no sub-expression in an occurrence of the Liar sentence (including 'true') is assessment sensitive (Ibid., 101). Simmons (2018b, 23, 36) states that his account-previously classified by himself and others as contextualist - treats 'true' as assessment sensitive. He takes this to be the case due to the arguments of Sagi 2017 (Ibid., fn. 24), and because 'its semantic value depends on the schema by which it is evaluated' (Ibid., 23). In order to meet MacFarlane's criteria to count as assessment sensitive (see Sect. 2.1), the extension of (non-subscripted) occurrences of 'true', and the relevant truth schema used for evaluation, would need to depend on the context of assessment. Yet Simmons (35-6) indicates that the issue of whether or not the extension of 'true' includes $L$ - which he takes to be a sentence type in a context-depends exclusively on the context of use for 'true'.
} 
contextualism (e.g., in Scharp 2013a, 71-3), the discovery that it entails his own position would be striking.

\section{Liar Contextualism Does Not Entail Relativism}

Having described the differences between contextualism and relativism, we are in a position to consider Sagi's (2017) view that Liar contextualism is committed to relativism. Section 3.1 sets out Sagi's main argument. I then present several objections. First, the values that Sagi takes to be assigned at different contexts of assessment in the course of the Liar argument do not seem to be genuinely conflicting (Sect. 3.2). Second, a close examination of a version of Liar contextualism that treats the domain of propositions as context dependent indicates that this type of account is not a form of relativism (Sect. 3.3). Finally, not even the weaker view that contextualist accounts are committed to a 'broad' form of relativism can be defended (Sect. 3.4).

\subsection{Sagi's General Argument}

The first question to address concerns what Sagi takes 'relativism' to be. She begins with several preliminary definitions (917-8). First, she considers an utterance $\alpha$ to be a pair consisting of a sentence $s_{\alpha}$ and a context of use $c_{\alpha}$. An utterance $\beta$ is an assessment of an utterance $\alpha$ if $\beta$ assigns to $\alpha$ an outcome of a truth evaluation. ${ }^{4}$ Finally, $\beta$ is a true assessment of $\alpha$ if $\beta$ is true with respect to the context of use $c_{\beta}$ and the relevant context of assessment ( $c_{\beta}$ by default, according to Sagi), which is the case only if the value that $\beta$ assigns to $\alpha$ is the one that $\alpha$ receives as used in $c_{\alpha}$ and assessed from $c_{\beta}$. Then: ${ }^{5}$

Assessment sensitive (Sagi) An utterance $\alpha$ is assessment sensitive if $\alpha$ 's evaluation depends on the context of assessment. Specifically, if there are two true assessments $\beta$ and $\gamma$ of $\alpha$ that occur at distinct contexts of use, and the values $\beta$ and $\gamma$ assign to $\alpha$ are conflicting, then $\alpha$ is assessment sensitive.

Sagi (fn. 5) states that '[c]onflicting values must be distinct' but 'not all distinct values are conflicting', and that she 'will not define conflicting values, but will rather go case by case'. She also notes that a sentence $s$ is assessment sensitive if there is an utterance of $s$ that is assessment sensitive, and a sub-sentential expression

\footnotetext{
4 Sagi admits that this is 'a vague definition' (917). As examples of sentences that can be used to formulate assessments, she gives: ' $\alpha$ is (true / false / not true / defective / without truth conditions / without truth value)' and ' $\alpha$ expresses (a true / a false / no) proposition'.

5 Sagi's definition indicates that she is taking the truth predicate to apply to utterances, an approach to which MacFarlane $(2014,107)$ objects, on the grounds that 'the monadic predicate "true" in ordinary use is a predicate of propositions'. MacFarlane (Ibid.) notes that 'we can understand utterance truth as a technical notion, by saying that an utterance at $c_{1}$ is true (as assessed from $c_{2}$ ) just in case the sentence uttered is true as used at $c_{1}$ and assessed from $c_{2}$ '. Hence a treatment of propositions as truth bearers may be recovered from Sagi's definition and discussion by replacing occurrences of ' $u_{i}$ ' with occurrences of 'the content expressed by $s_{i}$ as used at $c_{i}$ (and assessed from $c_{j}$ )'.
} 
is assessment sensitive if an explanation of the assessment sensitivity of a sentence 'hinges on the occurrence of this expression' (918).

Sagi is then in a position to state her key claim (918): 'in the Liar scenario, sensitivity to context of use entails assessment sensitivity'. To demonstrate this, she presents a version of the Liar argument (largely following Simmons 2015) that incorporates the analysis proposed by Liar contextualists, where the context shifts in the course of the reasoning. $L_{u}$ is a Liar utterance, insofar as it is an occurrence of the Liar sentence as used at context $c_{u}$ :

\section{Liar argument (Sagi):}

(1) $L_{u}: L_{u}$ is not true.

(2) $L_{u}$ is defective.

(3) $L_{u^{\prime}}: L_{u}$ is not true.

(4) $L_{u^{\prime}}$ is true.

The failure to assign $L_{u}$ a semantic value leads to the inference that it is defective. The fact that it is defective leads to the inference that the Liar utterance is not true, where this inference results in an utterance $L_{u^{\prime}}$ of the Liar sentence at a new context $c_{u^{\prime}}$. Since this latter utterance seems to have been properly inferred, it is concluded that it is true. For Liar contextualists, there is no contradiction, since distinct values have been assigned to distinct occurrences of the same sentence. Yet Sagi thinks that this reasoning process entails the assessment sensitivity of the Liar utterance. Her core argument is as follows:

The assessments in (2) and (3) were both inferred, and are taken to be true in this reasoning [...] The said assessments give different semantic values to $\left[L_{u}\right]$ : 'defective' and 'not true'. Moreover, these values are conflicting [...] Being defective should be understood here as resisting both values 'true' and 'not true' $[\ldots]$ Thus, by our definitions, $\left[L_{u}\right]$ is assessment-sensitive.

(Sagi 2017, 919).

Sagi goes on to provide further arguments that the specific Liar contextualist accounts proposed by Simmons and Glanzberg are committed to relativism. In Sect. 3.2 , I raise a concern about her core argument, before describing and evaluating her further claims in later subsections.

\subsection{Objection 1: The Values Need Not be Conflicting}

Sagi herself draws attention to a difficulty that her argument faces: $L_{u}$ can only meet her criteria to be assessment sensitive if ' $L_{u}$ is defective' and ' $L_{u}$ is not true' assign conflicting values to $L_{u}$, but (3) only seems to follow from (2) if these assessments do not assign conflicting values. That is, there can only be grounds for inferring ' $L_{u}$ is not true' if this is entailed by ' $L_{u}$ is defective'. Sagi responds to this difficulty as follows: 
[T] he contextualist might reply that it is the context shift that makes this inference possible: 'defective' is in conflict with 'not true' when the latter is uttered in the initial context (denying that $[L]$ is 'not true' was part of the reasoning that lead [sic] to the conclusion that it is defective) but is consonant with 'not true' as uttered in a later context. One can then press and ask what warrants the inference from (2) to (3). Sticking to the main issue at hand, I shall give the contextualists this move without attempting to defend it, and see what it implies.

(Sagi 2017, 919)

The idea would seemingly be that ' $L_{u}$ is defective' and ' $L_{u}$ is not true' assign conflicting values when used at $c_{u}$ and consonant values as used at $c_{u^{\prime}}$. Yet as Sagi notes, it would remain unclear what sort of principle a Liar contextualist could endorse that would entail: if ' $L_{u}$ is defective' holds relative to $c_{u}$ and 'is defective' means 'resisting both values 'true' and 'not true" at $c_{u}$, then ' $L_{u}$ is not true' holds relative to $c_{u^{\prime}}$. Still, she allows the contextualist this move, and takes it to imply that 'the same utterance receives conflicting values by true assessments in different contexts [...] of assessment'.

Sagi's response is puzzling for several reasons. First, the difficulty of maintaining that ' $L_{u}$ is defective' and ' $L_{u}$ is not true' assign conflicting values relative to the original context is well-known in the literature (see Gauker 2006, 396). Hence the most promising formulations of Liar contextualism avoid the sort of move that Sagi grants. ${ }^{6}$ Second, the move that Sagi allows the Liar contextualist to make is crucial to Sagi's own argument for the assessment sensitivity of the Liar utterance. For it is only by granting that ' $L_{u}$ is defective' and ' $L_{u}$ is not true' assign conflicting values for at least some contexts that we might conclude that $L_{u}$ receives conflicting values in the course of the Liar argument. Yet, third, even if we grant the sort of move that Sagi sketches, it still does not follow that $L_{u}$ is assigned conflicting values. Suppose that ' $L_{u}$ is defective' and ' $L_{u}$ is not true' assign conflicting values when both used at $c_{u}$ and consonant values when both used at $c_{u^{\prime}}$. In order to determine whether lines (2) and (3) of her Liar argument assign conflicting values, we would need to establish whether ' $L_{u}$ is defective' as used at $c_{u}$ and ' $L_{u}$ is not true' as used at $c_{u^{\prime}}$ assign conflicting values in some absolute sense. Yet Sagi provides no reason to think that this is the case.

In sum, it is difficult to see how to defend the claim that ' $L_{u}$ is defective' and ' $L_{u}$ is not true' assign conflicting values to $L_{u}$ in Sagi's formulation of the Liar argument. Yet it is only if they assign conflicting values that the assessment sensitivity of the Liar utterance follows.

\footnotetext{
${ }^{6}$ For instance, Simmons $(2018 \mathrm{~b}, 93)$ holds that $L$ (a sentence type in a context) is pathological [i.e., defective] because it cannot be assessed by its associated $c_{L}$-schema', where he takes the ' $c_{L}$-schema' to be conditions for truth in the context in which $L$ is originally used. He continues: 'Since $L$ cannot be assessed by its associated $c_{L}$-schema, it is not true $c_{c_{L}}$ ' (Ibid.). This strongly suggests that, rather than counting as conflicting values, $L_{u}$ 's being defective entails its being not true relative to the original context $c_{u}$. Also, many contextualists formulate versions of the Liar argument that make no reference whatsoever to a notion of defectiveness (e.g., Glanzberg 2004; Murzi and Rossi 2018).
} 


\subsection{Objection 2: Contextualism About the Domain of Propositions is not Relativism}

It is common to distinguish between two types of Liar contextualist account (see Simmons 2018a, 756): those that give a contextualist analysis of the English truth predicate (e.g., Burge 1982; Koons 1992; Simmons 1993, 2018b) and those that take the domain of propositions to depend on the context (e.g., Barwise and Etchemendy 1987; Glanzberg 2001, 2004, 2006; Murzi and Rossi 2018; Parsons 1974). After presenting her general argument, Sagi attempts to show that a paradigm version of each type of account is committed to relativism. Since her argument pertaining to Simmons' version of the first type of account is very similar to her general argument, I turn now to her discussion of Glanzberg's version of the second type of account. I give a brief overview of Glanzberg's account, before showing that it involves no commitment to relativism.

Since most truth predications apply the truth predicate to a proposition that is presented via a sentence that expresses it, Glanzberg claims that predications of truth generally require the expression relation. According to this view, to say ' $s_{i}$ is true' is really to say 'the proposition expressed by the sentence $s_{i}$ in context $c_{i}$ is true', which has as its logical form something like ' $\exists$ p $\left(\operatorname{Expresses}\left(s_{i}, p, c_{i}\right) \wedge\right.$ True $(p))$ '. Similarly, Sagi's formulation of the Liar utterance as ' $L_{u}$ is not true' is properly analysed as ' $\neg \exists p\left(\operatorname{Expresses}\left(L, p, c_{u}\right) \wedge \operatorname{True}(p)\right)$ '. The logical forms of truth predications therefore include an existential quantifier. By proposing that the context influences the domain of propositions over which this existential quantifier ranges, the potential emerges for truth predications such as the Liar sentence to be sensitive to contexts.

Glanzberg (2004, 33-4) attributes to the Liar sentence $L$ the logical form depicted in (1) below. ' $\ulcorner L\urcorner$ ' is a name for $L$, 'Exp' is interpreted as a two-place version of the expression relation Exp that omits reference to contexts (since the Liar sentence contains no use-sensitive expressions like indexicals), and ' $\mathrm{Tr}$ ' is a truth predicate of propositions. In the initial steps of the Liar argument (omitted for current purposes), assuming that $L$ expresses some proposition $q$ leads to the contradiction that $q$ is true and $q$ is not true. By standard rules of deductive logic, it follows that the assumption that $L$ expresses a proposition is false, as stated in (2). But then, the logical form in (3) follows by standard rules of logic, where this logical form is just $L$. Having just given a proof of $L, L$ must be true, so $L$ must express a proposition, as stated in (4):

\section{Liar argument (Glanzberg):}

(1) $\quad L: \neg \exists p(\operatorname{Exp}(\ulcorner L\urcorner, p) \wedge \operatorname{Tr}(p))$.

(2) $\neg \exists p \operatorname{Exp}(\ulcorner L\urcorner, p)$.

(3) $\neg \exists p(\operatorname{Exp}(\ulcorner L\urcorner, p) \wedge \operatorname{Tr}(p))$.

(4) $\exists p \operatorname{Exp}(\ulcorner L\urcorner, p)$.

The truth of (2) requires that there is no proposition for $L$ to express, while the truth of (4) requires that there is one, an apparent contradiction that Glanzberg's Liar 
contextualist analysis seeks to explain. He claims that the context has shifted between steps (2) and (3), because (2) is the first point in the Liar reasoning where the Exp relation is rendered salient, since it is the first point that includes 'Exp' in an assertion without undischarged premises (Glanzberg 2004, 39). The salience of the Exp relation yields a context where speakers are able to assert propositions concerning semantic relations like Exp, hence the domain of propositions that may be expressed by speakers at the later context exceeds the domain for the earlier context. It follows that there is no proposition for $L$ to express relative to the initial context, but there is a proposition for it to express relative to the later context with its expanded domain.

Sagi proffers several related arguments purporting to show that Glanzberg's analysis of the Liar sentence involves 'a type of assessment sensitivity' (2017, 923). Her first argument is that step (4) must be taken to involve an assessment of the original use of the Liar sentence, and that a single utterance is therefore assigned conflicting values in steps (2) and (4):

[I]t cannot be that $[L]$, as uttered in the later context has a proposition to express. This would lead to contradiction, exactly as it would in the initial context. It is $[L]$, as uttered in the initial context, that finally obtains a proposition to express. This leads us to relativism, at least in the broad sense: the same utterance (i.e. sentence in context) receives conflicting semantic evaluations.

(Sagi 2017, 923)

This argument appears to misrepresent Glanzberg's view: it is $L$ as used in the later context that expresses a proposition relative to the later context. This becomes apparent from Glanzberg's observation $(2004,75)$ that the sentence in (3) follows from the conclusion stated in the previous context in (2) only if the interpretation of 'Exp' and 'Tr' remains fixed as the context shifts. We therefore need to formulate within the later context an 'internal interpretation' of $L$ as it was interpreted in the original context, $\widetilde{L}$, where the occurrences of 'Exp' and ' $T r$ ' are interpreted as they were at the original context. A more accurate representation of the logical forms in steps (2)-(4) would therefore be as follows (see Glanzberg 2004, 75 for the formal representation, which is here adapted and simplified):

\section{Liar argument (Glanzberg):}

(2') $\neg \exists p \operatorname{Exp}(\ulcorner L\urcorner, p)$.

(3’) $\tilde{L}: \neg \exists p(\operatorname{Exp}(\ulcorner L\urcorner, p) \wedge \operatorname{Tr}(p))$.

(4') $\exists p \operatorname{Exp}(\ulcorner\tilde{L}\urcorner, p)$.

When $\tilde{L}$ is interpreted relative to the later context, it expresses the true proposition that $L$ as used in the earlier context does not express a true proposition (Ibid., 76).

As Glanzberg $(2004,79)$ explains, this proposal does not lead to further contradiction. For the occurrence of ' $T r$ ' in $\widetilde{L}$ is interpreted relative to the later context as an 'internal truth relation' $\tilde{T} r$ that reflects how the truth predicate was 
interpreted in the earlier context, and which is distinct from the truth relation of the later context. Hence the conclusion in $\left(4^{\prime}\right)$ that $\tilde{L}$ expresses a proposition $p$ at the later context cannot be used to show that $p$ is true and $p$ is not true at the later context, because the truth predicate that occurs in $\tilde{L}$ is not interpreted as the genuine truth relation of the later context. ${ }^{7}$

Sagi's next argument appears to be that we can take the contexts of (2) and (3) to be different contexts of assessment for the initial use of $L$, and that doing so would mean that '[a]ccording to Glanzberg's own view [...] the same content (fixed by the initial context) is assessed twice and receives different values: 'defective' and 'does not express a true proposition" (925-6). First, Glanzberg could not possibly agree that the same content is assessed twice: for his view is that $L$ expresses no content as used at the context of (2). ${ }^{8}$ Perhaps Sagi means that the same utterance is assessed twice. Yet even if we take the statements in (2) and (3) to be assessments of $L$ as used in the initial context, these assessments seemingly fail to assign conflicting values in the manner required for assessment sensitivity: the statement that $L$ does not express a proposition as used in the initial context is intuitively compatible with-and, indeed, entails - the statement that $L$ does not express a true proposition as used in the initial context.

Sagi's final argument seems to be that, even if Glanzberg takes step (4) in his Liar argument to involve an assessment of $\tilde{L}$ as used in the later context, we could 'reformulate the discourse in a faithful manner' without a second use of $L$, and would then be unable to avoid concluding that a single use of $L$ fails to express a proposition when assessed relative to the context of (2) while expressing a proposition when assessed relative to the context of (4) (926). ${ }^{9}$ Yet the repetition of $L$ is not immaterial: it is only through having proven $\widetilde{L}$ in line (3) that one is able to infer that $\tilde{L}$ is true relative to the context of (3), which allows the inference in step (4) that $\tilde{L}$ expresses a proposition relative to the context of (3). If the repetition of $L$ in the later context of line (3) is essential to generating the Liar argument, then the statement in line (4) will always be able to target a distinct use of $L$ to that of the evaluation in (2).

In sum, Liar contextualists who hold that the domain of propositions is context dependent avoid any commitment to the assessment sensitivity of the Liar sentence.

\footnotetext{
7 Of course, a new Liar paradox could be generated in the later context, possibly by uttering the sort of sentence that has the logical form of $L$ in the original context (e.g., 'This sentence is not true'). If we were to interpret Sagi as intending to claim that a new Liar sentence uttered in the later context cannot express a proposition relative to that context without engendering further paradox, then her claim would be correct. Yet it is not this sort of new Liar sentence that is referred to in step (4).

${ }^{8}$ For the same reason, it is difficult to see how Glanzberg's view could count as nonindexical contextualism (see fn. 2), despite Sagi's explicitly classifying it as such (2017, 924). Glanzberg's view appears to be a version of indexical contextualism: different contents are expressed by the same sentence at different contexts of use. That is, the Liar sentence fails to express any content whatsoever as used at one context and expresses true content as used at another context.

9 Note that this would be content relativism rather than truth-value relativism (see fn. 2 ).
} 


\subsection{Objection 3: Liar Contextualism Does Not Entail 'Broad' Relativism}

At several points, Sagi implies that certain views that do not involve assessment sensitivity still count as relativism:

$[\mathrm{C}]$ ontextualism as presented here at the very least yields relativism in a very general sense, by which truth-value is not determined by a sentence, context of use and circumstance of evaluation.

[W] semantic theory, we still remain with a form of relativism: we have an utterance receiving two different truth values on two occasions-it is just that now we cannot point to the context of assessments as the additional parameter by which semantic value can be determined.

[E]ven if one were to reject the appeal to assessments in the object language, we still find here an implication of relativism in the broad sense, as contexts of use are still insufficient to determine semantic value.

These quotations indicate that she is distinguishing between some 'broad' form of relativism and assessment sensitivity, although the nature of the distinction is never elaborated. ${ }^{10}$

Given that both the recent literature and Sagi herself consistently use the term 'relativism' to describe the view that some expressions are assessment sensitive, it is difficult to see how a position that rejects this view could reasonably be described as 'relativism' in any sense. At the very least, Sagi owes a detailed explanation of the features that her notion of 'broad' relativism shares with genuine relativism. Yet my arguments in Sect. 3.2-3.3 indicate that Liar contextualist accounts are simply committed to the use sensitivity of the Liar sentence; hence such analyses appear to be perfectly ordinary varieties of contextualism for which no additional label is necessary.

\section{The Falsity of the Liar Sentence Does Not Entail Relativism}

I turn now to a consideration of Rudnicki and Łukowski's (2019) account of the Liar argument, along with their claim that their account is committed to relativism. Section 4.1 describes their proposal and the experimental results that they take to support it. In Sect. 4.2, I observe some problems with their empirical data. In Sect. 4.3, I explain why their account does not count as relativism.

\footnotetext{
10 One natural thought would be that Sagi classifies Liar contextualists like Glanzberg as advocates of nonindexical contextualism (see fn. 2), and is relying on the fact that several in the literature have described such a position as 'relativism' (see Scharp 2013a, 236). Yet Sagi states that '[n]onindexical contextualism is still short of relativism as I have used the term' (924). Furthermore, there is good reason to classify Glanzberg as an indexical contextualist (see fn. 8); and no one in the literature has described this position as 'relativism', since it supplies the standard analysis for a wide range of use-sensitive expressions (indexicals, gradable adjectives, etc.).
} 


\subsection{Rudnicki and Łukowski's Argument}

Rudnicki and Łukowski endorse a solution to the Liar paradox based on the virtual entailment principle that they attribute to medieval thinkers such as Buridan: 'any proposition "virtually" entails another proposition that claims the original proposition to be true (where the point of "virtuality" seems to be that the relevant consequence need not actually be formed)' (Klima 2008, 107). According to this view, an occurrence of the Liar sentence $L$ asserts that $L$ is not true at the same time as 'virtually entailing' that $L$ is true. Hence the Liar sentence yields a straightforward contradiction, and is accordingly false. Moreover, Buridan claimed that the conclusion that $L$ is false does not permit the inference that $L$ is true, because even though $L$ correctly states that $L$ is not true, there is a stronger criterion of truth for a self-referential sentence than for an ordinary sentence, namely that whatever it entails be true (Read 2002, 198). Since the statement of $L$ does not permit the inference that $L$ is not true and that $L$ is true, it cannot be inferred that $L$ is defective. The Liar paradox is therefore resolved by precluding the reasoning that leads to apparently contradictory conclusions, both in the implicit steps between Sagi's steps (1) and (2) and in the inference from step (3) to (4) (see Sect. 3.1).

The claim that the truth of a self-referential sentence requires the truth of its entailments is described by Read (2002) as 'clearly ad hoc in the worst possible way' (199), on the grounds that it introduces a 'supplement to the truth-conditions of just those propositions which induce paradox to prevent the contradiction from arising' (217). Rudnicki and Łukowski $(2019,8)$ observe that the virtual entailment principle is amenable to empirical testing, because it predicts that the brain will 'react to the Liar sentence like it reacts to false sentences'. They thus aim to provide experimental support for the view that occurrences of the Liar sentence are false, in order to supply independent motivation for Buridan's solution to the Liar paradox and mitigate the charge that it is ad hoc.

They begin by providing an overview of the technique that they employ. Neurons in the brain give off electrical fields, and the associated changes in voltage can be detected via electrodes placed on the scalp. An event-related brain potential (ERP) consists of the pattern of voltage variation that occurs during a period time-locked to some stimulus (Coles and Rugg 1996, 1-2), and certain types of pattern are widely thought to co-vary with certain types of cognitive processes. Łukowski $(2019,8)$ claim that Hagoort et al. 2004 show that 'false sentences cause a deeper negative signal $400 \mathrm{~ms}$ from the last word in a sentence' compared with true sentences, where this is known as the N400 effect.

Rudnicki and Łukowski accordingly designed an experiment where thirty participants silently read Polish sentences of the following types, presented wordby-word; note that an anomalous sentence is one that is semantically or pragmatically odd in a manner that transcends mere falsity:

Liar sentences (e.g., 'This sentence is false').

Truthteller sentences (e.g., 'This sentence is true').

Self-referential false sentences (e.g., 'This sentence is in German').

Self-referential true sentences (e.g., 'This sentence is in English'). 
Self-referential anomalous sentences (e.g., 'This sentence is wet').

Non-self-referential false sentences (e.g., 'The sun is a planet').

Non-self-referential true sentences (e.g., 'The sun is a star').

Non-self-referential anomalous sentences (e.g., 'The sun is a crime').

They measured ERPs time-locked to the final words of 267 sentences of these eight types. Their aim was to evaluate the proposal that the Liar sentence is processed like false sentences by testing the following experimental hypothesis: 'Reading the Liar sentences causes higher N400 amplitude than reading true sentences' (12). They report that the results of their experiment support this hypothesis, and that the ERPs elicited by Liar sentences and other false sentences are 'statistically identical' (Ibid.).

They conclude that their experiment 'provides evidence supporting the virtual entailment principle and suggests that the Liar paradox may be resolved with it' (14). Interestingly, they claim that the resulting view falls within 'the relativistic view on truth, where adjectives pertaining to the truth-value are assessmentsensitive and an agent is required to perceive them to assess them (MacFarlane 2014)' (Ibid.). They do not fully elaborate their reason for drawing this inference, other than stating that their experiment investigates a 'physiological correlate of the fact that someone perceives a sentence to be false' (Ibid.), and 'we need to accept relativism to study human perceptions' (15).

\subsection{Objection 1: Their Results Do Not Support Their Account}

I will argue that the experimental hypothesis that Rudnicki and Łukowski formulate is ill-suited to provide evidence in favour of their proposal. Moreover, their results preclude the testing of the experimental hypothesis required to assess their proposal, due to potential methodological issues.

While Rudnicki and Łukowski imply that the N400 effect is predominantly associated with false sentences, the literature does not support this view (see Barrett and Rugg 1990; Dudschig et al. 2016; Federmeier and Kutas 1999; Holcomb and McPherson 1994; Kutas and Hillyard 1980, 1984; Kutas et al. 1988, 2006; Nigam et al. 1992; Otten and van Berkum 2007; van Berkum et al. 1999, 2003; van Petten and Kutas 1990; Willems et al. 2008). Indeed, Kutas and Hillyard 1980 originally identified the N400 effect for anomalous sentences. The class of anomalous sentences includes many that have been classified by philosophers as semantically defective and devoid of a truth value, rather than false. ${ }^{11}$ Rudnicki and Łukowski's linking of the N400 effect to falsity is based on experiments in Hagoort et al. 2004, which compared the ERPs elicited by target words in false and anomalous sentences to those of true sentences. Hagoort et al. $(2004,439)$ reported an N400 effect for both false and anomalous sentences, where the effect 'was identical in onset and peak latency and was very similar in amplitude and topographic distribution';

\footnotetext{
11 For instance, most analyses of the subclass of anomalous sentences often called 'category mistakes' treat them as semantically undefined or meaningless (see Asher 2011; Drange 1966; Fodor and Katz 1963; Goddard and Routley 1973; Lappin 1981; Martin 1974; Ryle 1938; Thomason 1972; van Fraassen 1971).
} 
although they observed that the amplitude of the N400 effect was slightly greater for anomalous than for false sentences. Dudschig et al. 2016 report the same findings, while identifying a slightly earlier onset of N400 effects for anomalous sentences than for false ones when they used a 'new exploratory method' of temporal analysis (44). These experiments provide evidence that processing certain false or anomalous sentences elicits the same type of neural activity, potentially with some very subtle differences in degree or timing.

A balanced overview of the ERP literature thus cautions against testing the hypothesis that the Liar sentence is processed like a false sentence by testing whether it elicits N400 effects relative to true sentences. For the mere eliciting of N400 effects by the Liar sentence would be compatible with the main rival to Rudnicki and Łukowski's analysis, which classifies the Liar sentence as defective in its original context (e.g., Liar contextualist accounts). The experimental hypothesis that could provide support for Rudnicki and Łukowski's account over the main rival analysis would state: reading Liar sentences elicits N400 effects (relative to true sentences) that are significantly different in onset and amplitude to those elicited by anomalous sentences, without being significantly different from those elicited by false sentences.

One problem with such a hypothesis is that, as previously mentioned, any differences in the N400 effects elicited by false and anomalous sentences are very subtle. It might therefore be considered inadvisable to use ERP data to attempt to adjudicate between the two rival accounts. A second problem is that Rudnicki and Łukowski's presentation of their results prevents even an informal comparison of the N400 effects elicited by false, anomalous and Liar sentences. While their experimental stimuli included the two types of anomalous sentences mentioned in Sect. 4.1 (i.e., self-referential anomalous sentences like 'This sentence is wet', and non-self-referential ones like 'The sun is a crime'), they entirely omitted the results for the anomalous sentences. ${ }^{12}$ Their explanation of this omission is: 'Because [anomalous sentences] showed highly different latency of the negative deflection it is not possible to accurately assess their N400 component' (17). This difference in latency is extremely surprising, since 'N400 latency for visual words is generally quite stable in the face of experimental manipulations' (Kutas et al. 2006, 676). If the N400 effects that the literature leads us to expect for anomalous sentences could not be identified in Rudnicki and Łukowski's data, then this suggests that there may have been issues with the experimental design, execution or method of analysis. Such issues might affect the reliability of the results even for the sentence types for which they were able to carry out an analysis.

In sum, support for the experimental hypothesis that reading Liar sentences elicits higher N400 amplitude than reading true sentences would not equate to support for Rudnicki and Łukowski's proposal. They do not formulate the experimental

\footnotetext{
12 Their only comment on these results is that there was a negative wave 'starting at the same time as false sentences (190 ms), but lasting much longer and peaking around $600 \mathrm{~ms}$ ' (17). They do not state whether they take this to be related to the N400 component, which peaks around 400ms after the relevant event. Indeed, it is possible that they are describing the N400-700 component, which is a later negative wave generally distinguished from the $\mathrm{N} 400$ component and linked to separate neural processes (see Kutas et al. 2006, 673).
} 
hypothesis appropriate to provide support for their account over the rival view that the Liar sentence is defective in its original context. Moreover, their presentation of results precludes even an informal assessment of the appropriate experimental hypothesis, in addition to raising the possibility of broader methodological issues. For these reasons, their experiment does not justify the conclusion that the Liar sentence should be analysed as false or that the virtual entailment principle holds.

\subsection{Objection 2: Their Account is Not a Form of Relativism}

The reason that Rudnicki and Łukowski link their proposal to relativism is seemingly that assessors' perceptions of sentences and truth values play a central role in their experiment and the resulting analysis of the Liar sentence. They are correct to emphasise the role of perceived truth value, rather than objective truth value, in their experiment and proposal. That is, if a true occurrence of a sentence were to be presented to individuals who failed to understand it or considered it false, then ERP data for that sentence would be likely to correspond more closely to data elicited by other sentences that the individuals failed to understand as true rather than data elicited by other true occurrences of sentences. Hence ERP experiments could only provide evidence that individuals process the Liar sentence like a false sentence, rather than that the Liar sentence is false. Yet it should be clear from Sect. 2.1 that relativism is distinct from the idea that perceived truth values may diverge from objective truth values, or even that there are no objective truth values. Rudnicki and Łukowski's endorsement of the virtual entailment principle means that an occurrence of the Liar sentence inevitably receives the value false, whatever its perceived value. This indicates that the Liar sentence is insensitive to both contexts of use and contexts of assessment. Hence their proposal rejects both contextualism and relativism.

It follows that, even if empirical support were to be attained for Rudnicki and Łukowski's proposal via further experiments involving appropriate hypotheses, this would not count as empirical support for a relativist proposal. Their experimental work is therefore not relevant to the ongoing debate in linguistics and the philosophy of language about whether any natural language expressions are assessment sensitive.

\section{Solutions to the Liar Paradox Need Not Entail Relativism}

Suppose that one grants the objections raised in Sects. 3 and 4, and thus rejects the recent arguments for connecting solutions to the Liar paradox with relativism. One might nevertheless believe that those who develop accounts of the Liar sentence are committed to a relativist analysis of the monadic truth predicate used in natural language, for independent reasons. Sagi $(2017,920)$ mentions, in passing, what we might take to be the best independent argument for this commitment: 'MacFarlane points out that relativism about any expression entails relativism about truth' ${ }^{13}$ MacFarlane's comment on the topic is as follows:

\footnotetext{
${ }_{13}$ I am grateful to an anonymous reviewer for also emphasising this point.
} 
'[I]f the language can express any assessment-sensitive propositions, "true" will also be assessment sensitive, since if $p$ is assessment-sensitive, the proposition that $p$ is true must be assessment-sensitive too.'

(MacFarlane 2014, 93)

This view implies that, if a relativist analysis is required for any natural language expressions - say, for predicates of personal taste like 'delicious' - then the objectlanguage, monadic truth predicate is assessment sensitive; and it would seem to follow that the only viable analyses of the Liar sentence would be relativist analyses.

This argument is ultimately unconvincing. First, the thesis that natural languages contain any assessment-sensitive expressions is controversial and frequently resisted (see Sect. 2). Second, the presence of assessment-sensitive expressions in a language entails that the monadic truth predicate is assessment sensitive in an importantly limited sense. To see this, consider MacFarlane's suggested analysis of the monadic truth predicate: ${ }^{14}$

[T] he context-relativized truth predicate used in semantics [...] is not the ordinary truth predicate used in everyday talk-a monadic predicate that applies to propositions [...] The relativist (or nonindexical contextualist) can treat the monadic predicate "true" as just another predicate of the object language - the language for which she is giving a semantics. [...] "True" expresses the same property at every context of use-the property of being true. The extension of this property at a circumstance of evaluation $e$ is the set of propositions that are true at $e$.

(MacFarlane 2014, 93)

According to this analysis, neither the context of use nor the context of assessment for 'true' will affect the content expressed, which will invariably be a function from each circumstance $e$ to the set of propositions true at $e$. Moreover, neither the context of use nor the context of assessment will consistently fix the circumstance $e$ that gives the relevant extension for the occurrence of 'true'. Hence the monadic truth predicate does not meet the criteria to count as a consistently assessment-sensitive or use-sensitive expression. Whether an occurrence of 'true' is assessment sensitive, use sensitive or neither will depend entirely on the status of the expression (denoting a sentence, proposition or utterance) to which it applies. ${ }^{15}$

\footnotetext{
${ }^{14}$ As MacFarlane $(2014$, fn. 27, 93) notes, this is a naive analysis that does not take the semantic paradoxes into consideration.

15 For instance, suppose that indexical contextualism (see fn. 2) provides the correct analysis of 'delicious', such that: an occurrence of 'Liquorice is delicious' used at $c_{1}$ and assessed at $c_{2}$ will express the proposition $p_{1}$ that liquorice is pleasing to the tastes at $c_{1}$, and will hold iff $p_{1}$ is true at the circumstance $e_{1}$ that includes the world of $c_{1}$. Then an occurrence of 'It is true that liquorice is delicious' used at $c_{1}$ and assessed at $c_{2}$ will express the proposition that $p_{1}$ is true, and will hold iff $p_{1}$ is true at $e_{1}$. On the other hand, suppose that truth-value relativism provides the correct analysis of 'delicious', such that: an occurrence of 'Liquorice is delicious' used at $c_{1}$ and assessed at $c_{2}$ will express the proposition $p_{2}$ that liquorice is pleasing to the tastes at the relevant circumstance, and will hold iff $p_{2}$ is true at the circumstance $e_{2}$ that includes the world of $c_{1}$ and the tastes of $c_{2}$. Then an occurrence of 'It is true that liquorice is delicious' used at $c_{1}$ and assessed at $c_{2}$ will express the proposition that $p_{2}$ is true, and will hold iff $p_{2}$ is true at $e_{2}$. In the first scenario, the occurrence of 'It is true that liquorice is delicious' is use sensitive, because its content is determined by the tastes of the context of use. In the second scenario, the occurrence of 'It is true that liquorice is delicious' is assessment sensitive, because the circumstance at
} 
The assessment sensitivity of the monadic truth predicate for a language with assessment-sensitive expressions is thus importantly limited: some but not all occurrences of 'true' will be assessment sensitive, and any assessment-sensitive occurrence inherits its assessment sensitivity from another expression. Indeed, 'true' would not meet the criteria to count as an assessment-sensitive sub-sentential expression according to Sagi's usage (see Sect. 3.1): an explanation of the assessment sensitivity of a sentence would never 'hinge' on the occurrence of the truth predicate. This contrasts with a monadic truth predicate that receives its own relativist analysis, such as the one proposed by Scharp $(2013 \mathrm{a}, \mathrm{b}):^{16}$

The specifically relativist feature of the semantics is that the points of evaluation have an additional parameter, which I call the aletheic standard. [...] The aletheic standard provides a "reading" of the occurrences of the truth predicate in the sentence in question. There are two possibilities for the aletheic standard parameter: the ascending standard $\left(\mathrm{S}_{A}\right)$ and the descending standard $\left(\mathrm{S}_{D}\right)$. The ascending standard interprets the truth predicate as an ascending truth predicate, while the descending standard treats the truth predicate as a descending truth predicate.

(Scharp 2013b, 464)

For current purposes, the difference between ascending and descending aletheic standards does not matter. The point is that, for any occurrence of the monadic truth predicate, the circumstance at which it is evaluated will include the aletheic standard of the context of assessment. Moreover, the extension of a use of 'true' may diverge relative to different contexts of assessment: the extension relative to $c_{1}$ might be the set of propositions that are ascending true at $w_{1}$, and the extension relative to $c_{2}$ might be the distinct set of propositions that are descending true at $w_{1}$. This analysis means that every occurrence of the monadic truth predicate will be inherently assessment sensitive, whatever the status of the expression to which it applies.

While the appeal of this analysis of 'true' might be questioned, the crucial point for current purposes is that such an analysis is not simply entailed by the presence of assessment-sensitive expressions. ${ }^{17}$ The presence of assessment-sensitive expressions could be said to entail a relativist analysis of the truth predicate only to the extent that the presence of use-sensitive expressions and context-insensitive expressions commits us to a simultaneously contextualist and context-invariant

\footnotetext{
Footnote 15 continued

which it is evaluated includes the tastes of the context of assessment. In both cases, it is the status of 'Liquorice is delicious' that determines the status of 'true'.

${ }^{16}$ One subtlety of Scharp's view is that his proposed analysis of the monadic truth predicate is not intended to capture how ordinary speakers actually use and interpret it, but how theorists should interpret it in order to resolve the semantic paradoxes (see Scharp 2013b, 492-3).

17 Scharp (2013b, 488) mentions some problems for such an analysis: 'The way aletheic standards work in the theory described above differs from the way most standards work in semantic relativist theories, and the arguments given for the theories above differ from the kind of evidence usually marshaled in favor of semantic relativist treatments'.
} 
analysis of the truth predicate: some but not all occurrences of 'true' inherit use sensitivity or assessment sensitivity from another expression. ${ }^{18}$

A third reason that the argument is unconvincing is that the occurrence of the truth predicate in the Liar sentence is assessment sensitive only if a Scharp-style relativist analysis is endorsed. For there is no candidate for an assessment-sensitive expression in the Liar sentence from which 'true' could inherit assessment sensitivity. The only other overt expressions in Liar sentences-a negation and a name for a particular sentence, utterance or proposition-are normally analysed as context invariant. The covert elements of Liar sentences that theorists have postulated - such as an existential quantifier ranging over propositions (see Sect. 3.3) - are analysed as use sensitive. Hence the presence of assessment-sensitive expressions in natural language would be insufficient to entail the assessment sensitivity of the occurrence of the truth predicate in the Liar sentence, or of the Liar sentence itself. Even if viable analyses of the truth predicate must be relativist in the limited sense of conceding the potential for assessment-sensitive occurrences, viable analyses of the Liar sentence need not be relativist in any sense.

\section{Conclusion}

There would be a significant impact on accounts of the Liar paradox if there were convincing arguments connecting such accounts to semantic relativism. If Sagi's arguments were to succeed, then Liar contextualist accounts would be implicitly committed to relativism. If Rudnicki and Łukowski's arguments were to succeed, then contextualist accounts, along with other traditional accounts of the Liar paradox, would have to address the empirical support available for a relativist analysis of the Liar sentence. Moreover, if the best independent argument for connecting the Liar paradox with relativism succeeds, then only relativist analyses of the Liar sentence would accurately capture the status of the monadic truth predicate. However, I have argued that contextualist accounts are not committed to relativism, and that there is no empirical evidence motivating an analysis of the Liar sentence that classifies it as false or accepts relativism. Since the best independent argument also fails, there is no reason to think that solutions to the Liar paradox require relativism. ${ }^{19}$

Open Access This article is licensed under a Creative Commons Attribution 4.0 International License, which permits use, sharing, adaptation, distribution and reproduction in any medium or format, as long as you give appropriate credit to the original author(s) and the source, provide a link to the Creative Commons licence, and indicate if changes were made. The images or other third party material in this article are included in the article's Creative Commons licence, unless indicated otherwise in a credit line to the material. If material is not included in the article's Creative Commons licence and your intended

\footnotetext{
18 Note that existing Liar contextualist take truth predications to be inherently use sensitive, and deny that the truth predicate in the Liar sentence is use sensitive solely by virtue of its application to a usesensitive expression.

19 For valuable discussion and comments, I am grateful to Christopher Gauker, Michael Glanzberg, John MacFarlane, Julien Murzi, Ashley Shaw, Johannes Stern, and the anonymous reviewers for this journal. Work on this article was supported by the European Research Council (ERC) Starting Grant Truth and Semantics (TRUST, Grant no. 803684).
} 
use is not permitted by statutory regulation or exceeds the permitted use, you will need to obtain permission directly from the copyright holder. To view a copy of this licence, visit http:// creativecommons.org/licenses/by/4.0/.

\section{References}

Asher, N. (2011). Lexical Meaning in Context: A Web of Words. Cambridge: Cambridge University Press. Barrett, S. E., \& Rugg, M. D. (1990). Event-related potentials and the semantic matching of pictures. Brain and Cognition, 14(2), 201-212.

Barwise, J., \& Etchemendy, J. (1987). The Liar. Oxford: Oxford University Press.

Beddor, B., \& Egan, A. (2018). Might do better: Flexible relativism and the QUD. Semantics and Pragmatics, 11(7), 1.

Billon, A. (2012). My own truth. In S. Rahman, G. Primiero, \& M. Marion (Eds.), The RealismAntirealism Debate in the Age of Alternative Logics (pp. 25-45). Dordrecht: Springer.

Bobzien, S. (2017). Gestalt shifts in the liar or why KT4M is the logic of semantic modalities. In B. Armour-Garb (Ed.), Reflections on the Liar (Vol. 1, pp. 71-113). Oxford: Oxford University Press.

Burge, T. (1982). The liar Paradox: Tangles and chains. Philosophical Studies, 41(3), 353-366.

Cappelen, H., \& Hawthorne, J. (2009). Relativism and Monadic Truth. Oxford: Oxford University Press.

Chihara, C. (1979). The semantic paradoxes: A diagnostic investigation. Philosophical Review, 88, $590-618$.

Coles, M. G. H., \& Rugg, M. D. (1996). Event-related brain potentials: An introduction. In M. D. Rugg \& M. G. H. Coles (Eds.), Electrophysiology of Mind (pp. 1-26). Oxford: Oxford University Press.

Drange, T. (1966). Type Crossings: Sentential Meaninglessness in the Border Area of Linguistics and Philosophy. The Hague: Mouton.

Dudschig, C., Maienborn, C., \& Kaup, B. (2016). Is there a difference between stripy journeys and stripy ladybirds? the N400 response to semantic and world-knowledge violations during sentence processing. Brain and Cognition, 103, 38-49.

Egan, A. (2007). Epistemic modals, relativism and assertion. Philosophical Studies, 133(1), 1-22.

Egan, A., Hawthorne, J., \& Weatherson, B. (2005). Epistemic modals in context. In G. Preyer \& G. Peter (Eds.), Contextualism in Philosophy (pp. 131-170). Oxford: Oxford University Press.

Federmeier, K. D., \& Kutas, M. (1999). A rose by any other name: Long-term memory structure and sentence processing. Journal of memory and Language, 41(4), 469-495.

Fodor, J. A., \& Katz, J. J. (1963). The structure of a semantic theory. Language, 39(2), 170-210.

Gauker, C. (2006). Against stepping back: A critique of contextualist approaches to the semantic paradoxes. Journal of Philosophical Logic, 35(4), 393-422.

Glanzberg, M. (2001). The liar in context. Philosophical Studies, 103(3), 217-251.

Glanzberg, M. (2004). A contextual-hierarchical approach to truth and the liar paradox. Journal of Philosophical Logic, 33(1), 27-88.

Glanzberg, M. (2006). Context and unrestricted quantification. In A. Rayo \& G. Uzquiano (Eds.), Absolute Generality (pp. 45-74). Oxford: Oxford University Press.

Glanzberg, M. (2007). Context, content, and relativism. Philosophical Studies, 136(1), 1-29.

Goddard, L., \& Routley, R. (1973). The Logic of Significance and Context. Edinburgh: Scottish Academic Press.

Gupta, A. (1982). Truth and paradox. Journal of Philosophical Logic, 11, 1-60.

Hagoort, P., Hald, L., Bastiaansen, M., \& Petersson, K. M. (2004). Integration of word meaning and world knowledge in language comprehension. Science, 304(5669), 438-441.

Holcomb, P. J., \& McPherson, W. B. (1994). Event-related brain potentials reflect semantic priming in an object decision task. Brain and Cognition, 24(2), 259-276.

Kaplan, D. (1989). Demonstratives. In J. Almog, J. Perry, \& H. Wettstein (Eds.), Themes from Kaplan (pp. 481-614). Oxford: Oxford University Press.

Katz, J., \& Salerno, J. (2017). Epistemic modal disagreement. Topoi, 36(1), 141-153.

Kennedy, C. \& McNally, L. (2005). Scale structure, degree modification, and the semantics of gradable predicates. Language, 81.

Khoo, J., \& Knobe, J. (2018). Moral disagreement and moral semantics. Noûs, 52(1), 109-143. 
Klima, G. (2008). Logic without truth. In S. Rahman, T. Tulenheimo, \& E. Genot (Eds.), Unity, Truth and the Liar: The Modern Relevance of Medieval Solutions to the Liar Paradox (pp. 87-112). New York, NY: Springer.

Knobe, J., \& Yalcin, S. (2014). Epistemic modals and context: Experimental data. Semantics and Pragmatics, 7(10), 1-21.

Kolodny, N., \& MacFarlane, J. (2010). Ifs and oughts. The Journal of Philosophy, 107(3), 115-143.

Koons, R. C. (1992). Paradoxes of Belief and Strategic Rationality. Cambridge: Cambridge University Press.

Kratzer, A. (1981). The notional category of modality. Words, Worlds, and Contexts: New Approaches in Word Semantics, 6, 38-74.

Kutas, M., \& Hillyard, S. A. (1980). Reading senseless sentences: Brain potentials reflect semantic incongruity. Science, 207(4427), 203-205.

Kutas, M., \& Hillyard, S. A. (1984). Brain potentials during reading reflect word expectancy and semantic association. Nature, 307(5947), 161-163.

Kutas, M., Van Petten, C. K., \& Besson, M. (1988). Event-related potential asymmetries during the reading of sentences. Electroencephalography and Clinical Neurophysiology, 69(3), 218-233.

Kutas, M., Van Petten, C. K., \& Kluender, R. (2006). Psycholinguistics electrified II (1994-2005). In M. J. Traxler \& M. A. Gernsbacher (Eds.), Handbook of Psycholinguistics (pp. 659-724). London: Academic Press.

Lappin, S. (1981). Sorts, Ontology, and Metaphor: The Semantics of Sortal Structure. De Gruyter, Berlin \& New York, NY.

Lasersohn, P. (2005). Context dependence, disagreement, and predicates of personal taste. Linguistics and Philosophy, 28, 643-686.

Lasersohn, P. (2009). Relative truth, speaker commitment, and control of implicit arguments. Synthese, 166(2), 359-374.

MacFarlane, J. (2003). Future contingents and relative truth. Philosophical Quarterly, 53(212), 321-336.

MacFarlane, J. (2005a). The assessment sensitivity of knowledge attributions. In T. S. Gendler \& J. Hawthorne (Eds.), Oxford Studies in Epistemology (pp. 197-233). Oxford: Oxford University Press.

MacFarlane, J. (2005b). Making sense of relative truth. Proceedings of the Aristotelian Society, 105, 321-339.

MacFarlane, J. (2007). Relativism and disagreement. Philosophical Studies, 132, 17-31.

MacFarlane, J. (2008). Truth in the garden of forking paths. In M. García-Carpintero \& M. Kölbel (Eds.), Relative Truth (pp. 81-102). Oxford: Oxford University Press.

MacFarlane, J. (2011a). Epistemic modals are assessment sensitive. In B. Weatherson \& A. Egan (Eds.), Epistemic Modality (pp. 144-178). Oxford: Oxford University Press.

MacFarlane, J. (2011b). Relativism and knowledge attributions. In D. Pritchard \& S. Bernecker (Eds.), The Routledge Companion to Epistemology (pp. 536-544). London: Routledge.

MacFarlane, J. (2011c). Simplicity made difficult. Philosophical Studies, 156(3), 441-448.

MacFarlane, J. (2012). Relativism. In G. Russell \& D. G. Fara (Eds.), The Routledge Companion to Philosophy of Language (pp. 132-142). New York: Routledge.

MacFarlane, J. (2014). Assessment Sensitivity: Relative Truth and Its Applications. Oxford: Oxford University Press.

Martin, R. L. (1974). Sortal ranges for complex predicates. Journal of Philosophical Logic, 3(1), 159-167.

Maudlin, T. (2004). Truth and Paradox: Solving the Riddle. Oxford: Oxford University Press.

McGee, V. (1991). Truth, Vagueness, and Paradox: An Essay on the Logic of Truth. Indianapolis: Hackett Publishing Company.

Murzi, J., \& Rossi, L. (2018). Reflection principles and the liar in context. Philosophers' Imprint, 18(15), $1-18$.

Nigam, A., Hoffman, J. E., \& Simons, R. F. (1992). N400 to semantically anomalous pictures and words. Journal of Cognitive Neuroscience, 4(1), 15-22.

Otten, M., \& van Berkum, J. J. (2007). What makes a discourse constraining? Comparing the effects of discourse message and scenario fit on the discourse-dependent N400 effect. Brain Research, 1153, 166-177.

Parsons, C. (1974). The liar paradox. Journal of Philosophical Logic, 3(4), 381-412.

Read, S. (2002). The liar paradox from John Buridan back to Thomas Bradwardine. Vivarium, 40(2), 189-218.

Richard, M. (2004). Contextualism and relativism. Philosophical Studies, 119(1/2), 215-242. 
Rudnicki, K., Łukowski, P. (2019). Psychophysiological Approach to the Liar Paradox: Jean Buridan's Virtual Entailment Principle Put to the Test. Synthese, pp. 1-20.

Ryle, G. (1938). Categories. Proceedings of the Aristotelian Society, 38, 189-206.

Sagi, G. (2017). Contextualism, relativism and the liar. Erkenntnis, 82(4), 913-928.

Scharp, K. (2013a). Replacing Truth. Oxford: Oxford University Press.

Scharp, K. (2013b). Truth, the liar, and relativism. Philosophical Review, 122(3), 427-510.

Shirreff, P., \& Weatherson, B. (2017). Relativism. In B. Hale, C. Wright, \& A. Miller (Eds.), A Companion to the Philosophy of Language (pp. 787-803). Chichester: Wiley.

Simmons, K. (1993). Universality and the Liar: An Essay on Truth and the Diagonal Argument. Cambridge: Cambridge University Press.

Simmons, K. (2015). Paradox, repetition, revenge. Topoi, 34(1), 121-131.

Simmons, K. (2018a). Contextual theories of truth and paradox. In M. Glanzberg (Ed.), The Oxford Handbook of Truth (Vol. 1, pp. 755-786). Oxford: Oxford University Press.

Simmons, K. (2018b). Semantic Singularities: Paradoxes of Reference, Predication, and Truth (1st ed.). Oxford: Oxford University Press.

Stanley, J., \& Szabó, Z. G. (2000). On quantifier domain restriction. Mind \& Language, 15(2-3), 219-261.

Stephenson, T. (2007). Judge dependence, epistemic modals, and predicates of personal taste. Linguistics and Philosophy, 30(4), 487-525.

Sundell, T. (2011). Disagreements about taste. Philosophical Studies, 155(2), 267-288.

Thomason, R. H. (1972). A semantic theory of sortal incorrectness. Journal of Philosophical Logic, 1(2), 209-258.

van Berkum, J. J. A., Hagoort, P., \& Brown, C. M. (1999). Semantic integration in sentences and discourse: evidence from the N400. Journal of Cognitive Neuroscience, 11(6), 657-671.

van Berkum, J. J. A., Zwitserlood, P., Hagoort, P., \& Brown, M. (2003). When and how do listeners relate a sentence to the wider discourse? Evidence from the N400 effect. Cognitive Brain Research, 17(3), 701-718.

van Fraassen, B. C. (1971). Formal Semantics and Logic. New York: Macmillan.

van Petten, C. K., \& Kutas, M. (1990). Interactions between sentence context and word frequency in event-related brain potentials. Memory \& Cognition, 18(4), 380-393.

Willems, R. M., Özyürek, A., \& Hagoort, P. (2008). Seeing and hearing meaning: ERP and fMRI evidence of word versus picture integration into a sentence context. Journal of Cognitive Neuroscience, 20(7), 1235-1249.

Publisher's Note Springer Nature remains neutral with regard to jurisdictional claims in published maps and institutional affiliations. 